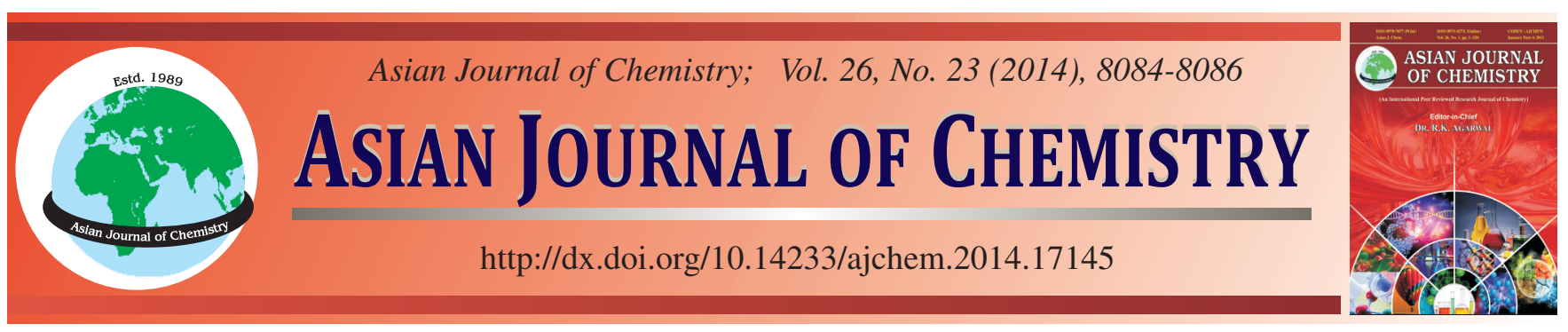

\title{
Reaction of Heteroaryl Hydrazines with 3-Cinnamoyl-4-hydroxy-6-methyl-2H- pyran-2-ones: Synthesis of Some Novel Pyrazolines and their Iodine(III) Mediated Conversion to Corresponding Pyrazoles
}

Mayank Kinger ${ }^{*}$ V. D. Gupta, Joginder Singh, Avnish Kumar Arora and Vivek Sheel Jaswal

Depatment of Chemistry, Maharishi Markandeshwar University, Mullana, Ambala-133 207, India

*Corresponding author: Fax: +91 1731 274375; Tel: +91 1731 304100; E-mail: mayank.kinger@yahoo.com

A series of some novel 5-aryl-3-(4-hydroxy-6-methyl-2H-pyran-2-oxo-3-yl)-1-heteroaryl pyrazolines have been prepared by the reaction of 3-cinnamoyl-4-hydroxy-6-methyl-2H-pyran-2-ones i.e., chalcone analogues of dehydroacetic acid (DHA) with various heteroaryl hydrazines and converted to corresponding pyrazoles with iodobenzene diacetate in good yields.

Keywords: Chalcone, Dehydroacetic acid, Heteroaryl hydrazines, Pyrazolines, Iodobenzene diacetate, Pyrazoles.

\section{INTRODUCTION}

Pyrazoles are one of the most important classes of compounds among heterocycles. A great deal of attention has been devoted to the chemistry of pyrazoles due to varied pharmacological applications such as CNS depressant, neuroleptic, tuberculostatic, antihypertensive, antileishmanial, analgesic, antidiabetic, antitumor, antimicrobial, antipyretic ${ }^{1-7}$. Pyrazole plays an important role in synthetic as well as medicinal chemistry as they constitute the framework of numerous drugs and dyes. Efforts are still continuously being made to evolve newer and facile procedures for the synthesis of this class of compounds. Among a number of methods reported in literature, one involving oxidation of pyrazolines with various oxidizing agents such as manganese dioxide, colloidal platinum, mercuric acetate, mercuric oxide and lead tetra- acetate, lead oxide etc., finds significant use in this area ${ }^{8,9}$. Thus there is a great demand for developing newer methods involving non-toxic oxidizing agents to effect such conversions.

Recent publications from our lab have emphasized the advantageous use of versatile organohypervalent iodine(III) reagents such as iodobenzene diacetate, hydroxytosyloxy iodobenzene (HTIB) etc., over toxic oxidizing reagents such as $\mathrm{Pb}(\mathrm{IV}), \mathrm{Tl}(\mathrm{III})$, or $\mathrm{Hg}(\mathrm{III})$ salts $^{10}$. Relevant to the present study on the oxidation of pyrazolines with iodobenzene diacetate to the pyrazoles ${ }^{11,12}$.

In literature, the reaction of phenyl and 6-fluorobenzothiazolyl hydrazines with various 1,3-diarylprop-2-en-1-ones have been studied and obtained pyrazolines were successfully dehydrogenated in to pyrazoles using iodobenzene diacetate ${ }^{11,12}$. But effect of the nature of heterocyclic moiety on the reaction of hydrazines with 3-cinnamoyl-4-hydroxy-6-methyl- $2 \mathrm{H}$ pyran-2-ones i.e., chalcone analogues of dehydroacetic acid particularly having pyran moiety has not been explored yet.

Prompted by these observations, it was thought of interest to investigate the reaction of heteroaryl hydrazines with 3-cinnamoyl-4-hydroxy-6-methylpyran-2-ones (3a-f) and to further study the iodine(III) mediated oxidative approach for the transformation of 5-aryl-3-(4-hydroxy-6-methyl-2Hpyran-2-oxo-3-yl)-1-hetarylpyrazolines (7a-f) into the corresponding 5-aryl-3-(4-hydroxy-6-methyl-2H-pyran-2-oxo-3yl)-1-hetarylpyrazoles (8a-f).

\section{EXPERIMENTAL}

Melting points were determined in open capillaries and are uncorrected. ${ }^{1} \mathrm{H}$ NMR spectra were recorded on Bruker $300 \mathrm{MHz}$ spectrophotometer. The internal standard used for these spectra was TMS. IR spectra $(\mathrm{KBr})$ were recorded on a Bruker-Vector 22 instrument. The stationary phases used for column chromatography (Silica gel 60, 70-230 mesh) and TLC plates (Silica-gel $60 \mathrm{~F}_{254}$ ) were purchased form Merck KGaA. All the chemicals were purchased from commercial suppliers and used without further purification.

General procedure for the synthesis of 5-aryl-3-(4hydroxy-6-methyl-2H-pyran-2-oxo-3-yl)-1-(2-benzothiazolyl)pyrazolines (7a-f): A mixture of 3-cinnamoyl-4hydroxy-6-methyl-2-pyrones (3) (0.001 mol) and 2-hydrazinobenzothiazole $(\mathbf{6})(0.001 \mathrm{~mol})$ in ethanol $(25 \mathrm{~mL})$ was heated 
under reflux for $2 \mathrm{~h}$. The preparation of compound $\mathbf{3}$ was done according to literature procedure $\mathrm{r}^{13,14}$. The progress of the reaction was monitored by TLC. After completion, the excess of ethanol was distilled off under reduced pressure. On cooling the reaction mixture, the product which separated out was filtered, washed with ethanol, dried and recrystallized from ethanol to afford compound $7 \mathbf{a}-\mathbf{f}$ in excellent yields.

5-Phenyl-3-(4-hydroxy-6-methyl-2H-pyran-2-oxo-3yl)-1-(2-benzothiazolyl)pyrazoline (7a): Yield $75 \%$; m.p. 188-190 ${ }^{\circ} \mathrm{C}$; IR $\left(\mathrm{KBr}, v_{\max }, \mathrm{cm}^{-1}\right)$ : 1728, $(\mathrm{C}=\mathrm{O})$; ${ }^{1} \mathrm{H}$ NMR $\left(\mathrm{CDCl}_{3}, 300 \mathrm{MHz}, \delta\right): 2.21\left(\mathrm{~s}, 3 \mathrm{H}, \mathrm{CH}_{3}\right), 3.65\left(\mathrm{dd}, 1 \mathrm{H}, \mathrm{C}_{4}-\mathrm{H}_{\mathrm{A}}\right.$, $J=17.7,5.2 \mathrm{~Hz}), 4.12\left(\mathrm{dd}, 1 \mathrm{H}, \mathrm{C}_{4}-\mathrm{H}_{\mathrm{B}}, J=17.7,10.1 \mathrm{~Hz}\right)$, $5.61\left(\mathrm{dd}, 1 \mathrm{H}, \mathrm{C}_{3}-\mathrm{H}, J=5.2,10.1 \mathrm{~Hz}\right), 6.04$ (s, 1H, C $5^{\prime}$-H, DHA), 7.26 (m, 9H, Ar), 13.52 (s, 1H, OH).

5-(4-Methylphenyl)-3-(4-hydroxy-6-methyl-2H-pyran2-oxo-3-yl)-1-(2-benzothiazolyl)pyrazoline (7b): Yield $78 \%$; m.p. $238-240{ }^{\circ} \mathrm{C}$; IR (KBr, $\left.v_{\max }, \mathrm{cm}^{-1}\right)$ : $1725,(\mathrm{C}=\mathrm{O}) ;{ }^{1} \mathrm{H}$ NMR $\left(\mathrm{CDCl}_{3}, 300 \mathrm{MHz}, \delta\right): 2.30\left(\mathrm{~s}, 3 \mathrm{H}, \mathrm{CH}_{3}\right), 2.31\left(\mathrm{~s}, 3 \mathrm{H}, \mathrm{CH}_{3}\right)$, $3.73\left(\mathrm{dd}, 1 \mathrm{H}, \mathrm{C}_{4}-\mathrm{H}_{\mathrm{A}}, J=17.5,5.4 \mathrm{~Hz}\right), 4.18\left(\mathrm{dd}, 1 \mathrm{H}, \mathrm{C}_{4}-\mathrm{H}_{\mathrm{B}} J\right.$ $=17.5,11.1 \mathrm{~Hz}), 5.68\left(\mathrm{dd}, 1 \mathrm{H}, \mathrm{C}_{3}-\mathrm{H}, J=11.1,5.4 \mathrm{~Hz}\right), 6.08$ (s, 1H, C $\left.5^{\prime}-\mathrm{H}, \mathrm{DHA}\right), 7.27$ (m, 8H, Ar), 13.53 (s, 1H, OH).

5-(4-Chlorophenyl)-3-(4-hydroxy-6-methyl-2H-pyran2-oxo-3-yl)-1-(2-benzothiazolyl)pyrazoline (7c): Yield $76 \%$; m.p. $198-200{ }^{\circ} \mathrm{C}$; IR $\left(\mathrm{KBr}, \mathrm{v}_{\max }, \mathrm{cm}^{-1}\right)$ : $1723,(\mathrm{C}=\mathrm{O})$; ${ }^{1} \mathrm{H}$ NMR $\left(\mathrm{CDCl}_{3}, 300 \mathrm{MHz}, \delta\right): 2.28\left(\mathrm{~s}, 3 \mathrm{H}, \mathrm{CH}_{3}\right), 3.68\left(\mathrm{dd}, 1 \mathrm{H}, \mathrm{C}_{4}-\mathrm{H}_{\mathrm{A}}\right.$, $J=17.2,5.3 \mathrm{~Hz}), 4.19\left(\mathrm{dd}, 1 \mathrm{H}, \mathrm{C}_{4}-\mathrm{H}_{\mathrm{B}}, J=17.2,10.5 \mathrm{~Hz}\right)$, $5.65\left(\mathrm{dd}, 1 \mathrm{H}, \mathrm{C}_{3}-\mathrm{H}, J=10.5,5.3 \mathrm{~Hz}\right), 6.02\left(\mathrm{~s}, 1 \mathrm{H}, \mathrm{C}_{5^{\prime}}-\mathrm{H}, \mathrm{DHA}\right)$, 7.47 (m, 8H, Ar), 13.52 (s, 1H, OH).

5-(2-Methoxyphenyl)-3-(4-hydroxy-6-methyl-2Hpyran-2-oxo-3-yl)-1-(2-benzothiazolyl)pyrazoline (7d): Yield $79 \%$, m.p. $246-248^{\circ} \mathrm{C}$; IR $\left(\mathrm{KBr}, v_{\max }, \mathrm{cm}^{-1}\right)$ : $1725,(\mathrm{C}=\mathrm{O})$; ${ }^{1} \mathrm{H} \mathrm{NMR}\left(\mathrm{CDCl}_{3}, 300 \mathrm{MHz}, \delta\right): 2.20\left(\mathrm{~s}, 3 \mathrm{H}, \mathrm{CH}_{3}\right), 3.85$ (s, 3H, $\left.\mathrm{CH}_{3}\right), 3.51\left(\mathrm{dd}, 1 \mathrm{H}, \mathrm{C}_{4}-\mathrm{H}_{\mathrm{A}}, J=17.4,5.5 \mathrm{~Hz}\right), 4.07$ (dd, $1 \mathrm{H}, \mathrm{C}_{4}-$ $\left.\mathrm{H}_{\mathrm{B}}, J=17.4,10.6 \mathrm{~Hz}\right), 5.84\left(\mathrm{dd}, \mathrm{C}_{3}-\mathrm{H}, J=10.6,5.5 \mathrm{~Hz}\right), 6.08$ (s, 1H, C $\left.5^{\prime}-\mathrm{H}, \mathrm{DHA}\right), 7.28$ (m, 8H, Ar), 13.52 (s, 1H, OH).

5-(4-Fluorophenyl)-3-(4-hydroxy-6-methyl-2H-pyran2-oxo-3-yl)-1-(2-benzothiazolyl)pyrazoline (7e): Yield $78 \%$; m.p. $228-230{ }^{\circ} \mathrm{C}$; IR $\left(\mathrm{KBr}, \mathrm{v}_{\max }, \mathrm{cm}^{-1}\right)$ : $1725,(\mathrm{C}=\mathrm{O})$; ${ }^{1} \mathrm{H}$ NMR $\left(\mathrm{CDCl}_{3}, 300 \mathrm{MHz}, \delta\right): 2.23\left(\mathrm{~s}, 3 \mathrm{H}, \mathrm{CH}_{3}\right), 3.63\left(\mathrm{dd}, 1 \mathrm{H}, \mathrm{C}_{4}-\mathrm{H}_{\mathrm{A}}\right.$, $J=17,5.6 \mathrm{~Hz}), 4.11\left(\mathrm{dd}, 1 \mathrm{H}, \mathrm{C}_{4}-\mathrm{H}_{\mathrm{B}}, J=17,10.6 \mathrm{~Hz}\right), 5.60$ (dd, $\left.1 \mathrm{H}, \mathrm{C}_{3}-\mathrm{H}, J=10.6,5.6 \mathrm{~Hz}\right), 6.08$ (s, 1H, $\left.\mathrm{C}_{5^{\prime}} \mathrm{H}, \mathrm{DHA}\right)$, 7.28 (m, 8H, Ar), 13.51 (s, 1H, OH).

5-(2-Chlorophenyl)-3-(4-hydroxy-6-methyl-2H-pyran2-oxo-3-yl)-1-(2-benzothiazolyl)pyrazoline (7f): Yield $76 \%$; m.p. $223-225^{\circ} \mathrm{C}$; IR $\left(\mathrm{KBr}, \mathrm{v}_{\max }, \mathrm{cm}^{-1}\right)$ : $1725,(\mathrm{C}=\mathrm{O})$; ${ }^{1} \mathrm{H}$ NMR $\left(\mathrm{CDCl}_{3}, 300 \mathrm{MHz}, \delta\right): 2.21\left(\mathrm{~s}, 3 \mathrm{H}, \mathrm{CH}_{3}\right), 3.54\left(\mathrm{dd}, 1 \mathrm{H}, \mathrm{C}_{4}-\mathrm{H}_{\mathrm{A}}\right.$, $J=17.4,5.6 \mathrm{~Hz}), 4.21\left(\mathrm{dd}, 1 \mathrm{H}, \mathrm{C}_{4}-\mathrm{H}_{\mathrm{B}}, J=17.4,10.8 \mathrm{~Hz}\right)$, $5.92\left(\mathrm{dd}, 1 \mathrm{H}, \mathrm{C}_{3}-\mathrm{H}, J=10.8,5.6 \mathrm{~Hz}\right), 6.08\left(\mathrm{~s}, 1 \mathrm{H}, \mathrm{C}_{5^{\prime}}-\mathrm{H}, \mathrm{DHA}\right)$, 7.27 (m, 8H, Ar), 13.51 (s, 1H, OH).

General procedure for the synthesis of 5-aryl-3-(4hydroxy-6-methyl-2H-pyran-2-oxo-3-yl)-1-(2-benzothiazolyl)-pyrazoles (8a-f): To a stirred solution of 7 (0.001 mol) in dichloromethane $(15 \mathrm{~mL})$ was added iodobenzene diacetate $(0.0012 \mathrm{~mol})$ at room temperature. The reaction mixture was stirred for $5 \mathrm{~h}$. The progress of the reaction was monitored by TLC. Dichloromethane was distilled off on a steam bath and the residual gummy mass was triturated with petroleum ether to remove iodobenzene and was purified by recrystallization from ethanol to afford compound $\mathbf{8 a - f}$ in excellent yield.
5-Phenyl-3-(4-hydroxy-6-methyl-2H-pyran-2-oxo-3yl)-1-(2-benzothiazolyl)pyrazole (8a): Yield 72 \%; m.p. 188$190{ }^{\circ} \mathrm{C}$; IR $\left(\mathrm{KBr}, \mathrm{v}_{\max }, \mathrm{cm}^{-1}\right): 1728,(\mathrm{C}=\mathrm{O}) ;{ }^{1} \mathrm{H} \mathrm{NMR}\left(\mathrm{CDCl}_{3}\right.$, $300 \mathrm{MHz}, \delta$ ): 2.27 (s, 3H, $\mathrm{CH}_{3}$ ), 6.02 (s, 1H, C $\left.5^{-} \mathrm{H}, \mathrm{DHA}\right)$, 7.10 (s, 1H, C4-H), 7.28 (m, 9H, Ar), 13.54 (s, 1H, OH); Anal. found: $\mathrm{C}, 65.82 ; \mathrm{H}, 3.77 ; \mathrm{N}, 10.47 ; \mathrm{C}_{22} \mathrm{H}_{15} \mathrm{~N}_{3} \mathrm{O}_{3} \mathrm{~S}$ requires: $\mathrm{C}$, $65.80 ; \mathrm{H}, 3.74 ; \mathrm{N}, 10.49 \%$.

5-(4-Methylphenyl)-3-(4-hydroxy-6-methyl-2H-pyran2-oxo-3-yl)-1-(2-benzothiazolyl)pyrazole (8b): Yield $74 \%$; m.p. $213-215{ }^{\circ} \mathrm{C}$; IR $\left(\mathrm{KBr}, \mathrm{v}_{\max }, \mathrm{cm}^{-1}\right)$ : $1725,(\mathrm{C}=\mathrm{O})$; ${ }^{1} \mathrm{H}$ NMR $\left(\mathrm{CDCl}_{3}, 300 \mathrm{MHz}, \delta\right): 2.22$ (s, 3H, $\left.\mathrm{CH}_{3}\right), 2.41$ (s, 3H, $\left.\mathrm{CH}_{3}\right)$, 6.02 (s, 1H, C $\left.5^{\prime}-\mathrm{H}, \mathrm{DHA}\right), 7.32$ (s, 1H, C4-H), 7.28 (m, 8H, $\mathrm{Ar}), 13.53$ (s, 1H, OH); Anal. found: C, 66.49; H, 4.12; N, $10.11 ; \mathrm{C}_{23} \mathrm{H}_{17} \mathrm{~N}_{3} \mathrm{O}_{3} \mathrm{~S}$ requires: $\mathrm{C}, 66.43 ; \mathrm{H}, 4.09 ; \mathrm{N}, 10.19 \%$.

5-(4-Chlorophenyl)-3-(4-hydroxy-6-methyl-2H-pyran2-oxo-3-yl)-1-(2-benzothiazolyl)pyrazole (8c): Yield $74 \%$; m.p. $170-172{ }^{\circ} \mathrm{C}$; IR $\left(\mathrm{KBr}, \mathrm{v}_{\max }, \mathrm{cm}^{-1}\right)$ : $1723,(\mathrm{C}=\mathrm{O})$; ${ }^{1} \mathrm{H}$ NMR $\left(\mathrm{CDCl}_{3}, 300 \mathrm{MHz}, \delta\right): 2.29$ (s, 3H, $\left.\mathrm{CH}_{3}\right), 6.01$ (s, $1 \mathrm{H}, \mathrm{C}_{5^{\prime}} \mathrm{H}$, DHA), 7.42 (s, 1H, C 4 -H), 7.31 (m, 8H, Ar), 13.5 (s, 1H, OH); Anal. found: C, 60.62; H, 3.24; N, 9.69; $\mathrm{C}_{22} \mathrm{H}_{14} \mathrm{~N}_{3} \mathrm{O}_{3} \mathrm{SCl}$ requires: $\mathrm{C}, 60.67 ; \mathrm{H}, 3.21 ; \mathrm{N}, 9.72 \%$.

5-(2-Methoxyphenyl)-3-(4-hydroxy-6-methyl-2Hpyran-2-oxo-3-yl)-1-(2-benzothiazolyl)pyrazole (8d): Yield $78 \%$; m.p. $208-210{ }^{\circ} \mathrm{C}$; IR (KBr, $\left.v_{\max }, \mathrm{cm}^{-1}\right)$ : 1723, $(\mathrm{C}=\mathrm{O})$; ${ }^{1} \mathrm{H}$ NMR $\left(\mathrm{CDCl}_{3}, 300 \mathrm{MHz}, \delta\right): 2.22\left(\mathrm{~s}, 3 \mathrm{H}, \mathrm{CH}_{3}\right), 6.02$ (s, 1H, $\left.\mathrm{C}_{5^{\prime}} \mathrm{H}, \mathrm{DHA}\right), 7.41$ (s, 1H, C $\left.{ }_{4}-\mathrm{H}\right), 7.29$ (m, 8H, Ar), 13.5 (s, 1H, OH); Anal. found: $\mathrm{C}, 64.03 ; \mathrm{H}, 3.94 ; \mathrm{N}, 9.74$; $\mathrm{C}_{23} \mathrm{H}_{17} \mathrm{~N}_{3} \mathrm{O}_{4} \mathrm{~S}$ requires: $\mathrm{C}, 64.08 ; \mathrm{H}, 3.96 ; \mathrm{N}, 9.76 \%$.

5-(4-Fluorophenyl)-3-(4-hydroxy-6-methyl-2H-pyran2-oxo-3-yl)-1-(2-benzothiazolyl)pyrazole (8e): Yield $76 \%$; m.p. $178-179{ }^{\circ} \mathrm{C}$; IR $\left(\mathrm{KBr}, v_{\max }, \mathrm{cm}^{-1}\right)$ : $1723,(\mathrm{C}=\mathrm{O}) ;{ }^{1} \mathrm{H}$ NMR $\left(\mathrm{CDCl}_{3}, 300 \mathrm{MHz}, \delta\right): 2.23$ (s, 3H, $\left.\mathrm{CH}_{3}\right), 6.02$ (s, $1 \mathrm{H}, \mathrm{C}_{5^{\prime}} \mathrm{H}$, DHA), 7.39 (s, 1H, C4-H), 7.30 (m, 8H, Ar), 13.5 (s, 1H, OH); Anal. found: $\mathrm{C}, 63 ; \mathrm{H}, 3.36 ; \mathrm{N}, 10.02 ; \mathrm{C}_{22} \mathrm{H}_{14} \mathrm{~N}_{3} \mathrm{O}_{3}$ SF requires: C, 63.08; H, 3.39; N, $10.07 \%$.

5-(2-Chlorophenyl)-3-(4-hydroxy-6-methyl-2H-pyran2-oxo-3-yl)-1-(2-benzothiazolyl)pyrazole (8f): Yield $75 \%$; m.p. $165-167^{\circ} \mathrm{C}$; IR $\left(\mathrm{KBr}, v_{\max }, \mathrm{cm}^{-1}\right)$ : $1723,(\mathrm{C}=\mathrm{O}) ;{ }^{1} \mathrm{H}$ NMR $\left(\mathrm{CDCl}_{3}, 300 \mathrm{MHz}, \delta\right): 2.25$ (s, 3H, $\left.\mathrm{CH}_{3}\right), 6.01$ (s, $1 \mathrm{H}, \mathrm{C}_{5^{\prime}}-\mathrm{H}$, DHA), 7.38 (s, 1H, C4-H), 7.28 (m, 8H, Ar), 13.5 (s, 1H, OH); Anal. found: $\mathrm{C}, 60.62 ; \mathrm{H}, 3.24 ; \mathrm{N}, 9.64 ; \mathrm{C}_{22} \mathrm{H}_{14} \mathrm{~N}_{3} \mathrm{O}_{3} \mathrm{SCl}$ requires: $\mathrm{C}, 60.69 ; \mathrm{H}, 3.26 ; \mathrm{N}, 9.69 \%$.

\section{RESULTS AND DISCUSSION}

The 3-cinnamoyl-4-hydroxy-6-methylpyran-2-ones (3a-f) i.e., chalcone analogues of dehydroacetic acid (DHA) were prepared according to literature procedure by the reaction of dehydroacetic acid (1) with various aromatic aldehydes on refluxing in chloroform having few drops of piperidine for 6-8 $\mathrm{h}^{13,14}$ (Scheme-I). In order to prepare pyrazolines derivatives, the chalcones (3a-f) were treated with various heteroaryl hydrazines namely 2-hydrazino-4,6-dimethylpyrimidine (4), 2-hydrazino-4-methylquinoline (5) and 2-hydrazino-benzothiazole (6), in ethanol under reflux. Surprisingly, the reaction afforded the desired pyrazolines (7a-f) only in the case of 2hydrazinobenzothiazole (6). In other cases the starting materials were recovered, (Scheme-II). Repeated efforts by changing the reaction conditions did not show any significant improvement 


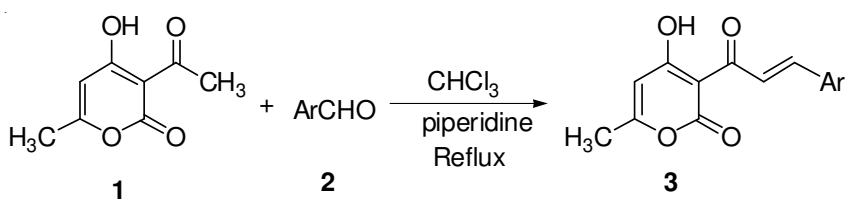

Scheme-I: Synthesis of 3-cinnamoyl-4-hydroxy-6-methyl-2H-pyran-2-ones (3a-f)

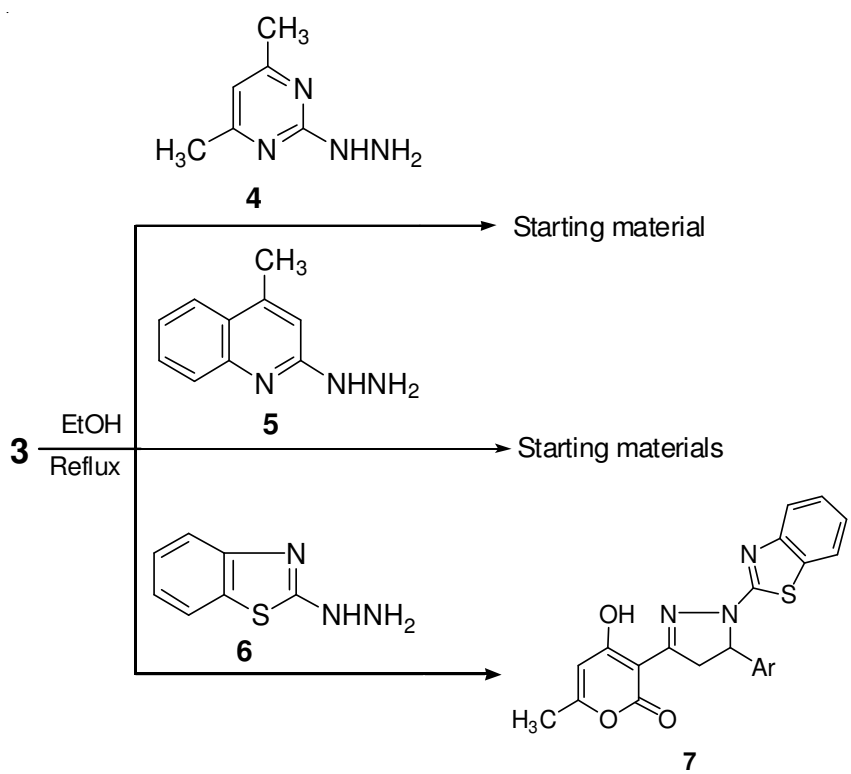

Scheme-II: Synthesis of 5-aryl-3-(4-hydroxy-6-methyl-2H-pyran-2-oxo-3yl)-1-hetarylpyrazolines (7a-f)

in the formation of desired or undesired products. Therefore further work was confined to the pyrazolines obtained from 2-hydrazinobenzothiazole.

Accordingly the pyrazoline derivatives 7a-f was prepared by the reaction between equimolar quantity of chalcones 3a-f and 2-hydrazinobenzothiazole (6) in ethanol (Scheme-II) and was characterized by spectral and analytical data.

Further to explore the use of hypervalent iodine(III) reagents, the oxidation of 5-aryl-3-(4-hydroxy-6-methylpyran2-oxo-3-yl)-1-(2-benzothiazolyl)pyrazolines (7a-f) were conducted by using 1.1 eq. of iodobenzene diacetate in $\mathrm{CH}_{2} \mathrm{Cl}_{2}$. The reaction occurred at room temperature smoothly to afford the corresponding 5-aryl-3-(4-hydroxy-6-methylpyran-2-oxo3-yl)-1-(2-benzothiazolyl)pyrazoles (8a-f) in excellent yield, (Scheme-III). Failure of the formation of pyrazolines from 2hydrazino-4,6-dimethylpyrimidine (4) and 2-hydrazino-4methylquinoline (5) did not allow us to carry out oxidation studies on such pyrazolines.

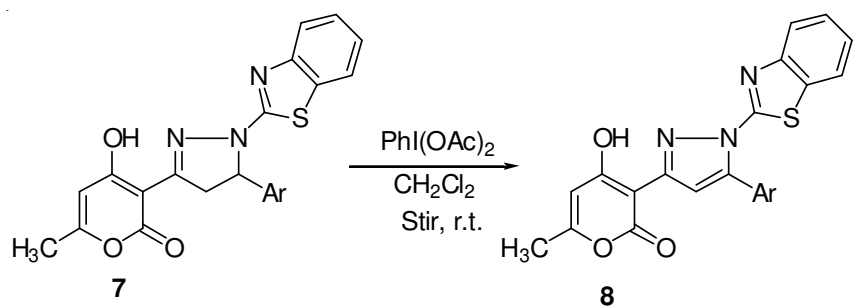

Scheme-III: Synthesis of 5-aryl-3-(4-hydroxy-6-methyl-2H-pyran-2-oxo3-yl)-1-hetarylpyrazoles (8a-f); 8a: Phenyl; 8b: 4Methylphenyl; 8c: 4-Chlorophenyl; 8d: 2-Methoxyphenyl; 8e: 4-Fluorophenyl; 8f: 2-Chlorophenyl
Confirmation of the occurrence of the transformation of pyrazolines 7a-f to pyrazoles 8a-f was based upon a careful comparison of $1 \mathrm{H}$ NMR spectra. The spectra of pyrazolines showed three characteristic signals due diastereotopic protons (HA, HB and HX). The proton which is cis to HX resonates upfield in the range of $\delta 3.63-3.72$ as a doublet of doublet (dd, $J_{\mathrm{HAHB}}=\sim 17.7, J_{\mathrm{HAHX}}=\sim 5.2 \mathrm{~Hz}$ ) while $\mathrm{HB}$ which is trans to HX resonates downfield in the range of $\delta 4.18-4.24$ as a dd $\left(\mathrm{dd}, J_{\mathrm{HAHB}}=\sim 17.7, J_{\mathrm{HBHX}}=\sim 10.1 \mathrm{~Hz}\right)$. The $\mathrm{HX}$ which is vicinal to two methylene protons $\left(\mathrm{H}_{\mathrm{A}}\right.$ and $\left.\mathrm{H}_{\mathrm{B}}\right)$ resonates as dd in the range of $\delta 5.62-5.68\left(\mathrm{dd}, J_{\mathrm{HAHX}}=\sim 5.2, J_{\mathrm{HBHX}}=\sim 10.1 \mathrm{~Hz}\right)$, respectively. On the other hand, the spectra of the corresponding oxidized products pyrazoles, which showed a characteristic singlet at around $\delta \sim 7.4$ due to $\mathrm{C} 4$ of the pyrazole, were devoid of all the characteristic signals of starting pyrazoline.

\section{Conclusion}

In conclusion, we have prepared a series of novel pyrazolines from the reaction of chalcone analogues of dehydroacetic acid with various heteroaryl hydrazines and successfully converted in to pyrazole using hypervalent iodine(III) mediated oxidative approach, thereby emphasizing further utility of iodine(III) reagents.

\section{ACKNOWLEDGEMENTS}

The authors are thankful to Maharishi Markandeshwar University, Mullana, India for financial assistance.

\section{REFERENCES}

1. K.S. Girisha, B. Kalluraya, V. Narayana and Padmashree, Eur. J. Med. Chem., 45, 4640 (2010).

2. B.S. Holla, M. Mahalinga, M.S. Karthikeyan, P.M. Akberali and N.S. Shetty, Bioorg. Med. Chem., 14, 2040 (2006).

3. F. Manna, F. Chimenti, R. Fioravanti, A. Bolasco, D. Secci, P. Chimenti, C. Ferlini and G. Scambia, Bioorg. Med. Chem. Lett., 15, 4632 (2005).

4. J.M. Fevig, J. Cacciola, J. Buriak Jr., K.A. Rossi, R.M. Knabb, J.M. Luettgen, P.C. Wong, S.A. Bai, R.R. Wexler and P.Y.S. Lam, Bioorg. Med. Chem. Lett., 16, 3755 (2006).

5. M.G.C. Kahn, E. Konde, F. Dossou, D.C. Labaree, R.B. Hochberg and R.M. Hoyte, Bioorg. Med. Chem. Lett., 16, 3454 (2006).

6. P. Cernuchová, G. Vo-Thanh, V. Milata, A. Loupy, S. Jantová and M. Theiszová, Tetrahedron, 61, 5379 (2005).

7. Z. Sui, J. Guan, M.P. Ferro, K. McCoy, M.P. Wachter, W.V. Murray, M. Singer, M. Steber, D.M. Ritchie and D.C. Argentieri, Bioorg. Med. Chem. Lett., 10, 601 (2000).

8. G.A. Golubeva, Ph. D. Thesis, Moscow University (1962).

9. I. Bhatnagar and M.V. George, Tetrahedron, 24, 1293 (1968).

10. O. Prakash, M. Kumar, R. Kumar, C. Sharma and K.R. Aneja, Eur. J. Med. Chem., 45, 4252 (2010).

11. O. Prakash, A. Kumar, M. Kinger and S.P. Singh, Indian J. Chem., 44B, 456 (2006).

12. R. Aggarwal, V. Kumar and S.P. Singh, Indian J. Chem., 46B, 1332 (2007).

13. R. Filler, V.D. Beaucaire and H.H. Kang, J. Org. Chem., 40, 935 (1975).

14. R.H. Wiley, C.H. Jarboe and H.G. Ellert, J. Am. Chem. Soc., 77, 5102 (1955). 